\title{
„Hägerstrand online“: Ein methodisches Konzept zur Analyse raumzeitlicher Trajektorien in Kollaborationen
}

\section{"Hägerstrand online": A methodical template for the analysis of space-time trajectories in collaborations}

https://doi.org/10.2478/rara-2019-0051

Eingegangen: 11. Oktober 2018; Angenommen: 6. August 2019

Kurzfassung: In der Wirtschaftsgeographie stieg in den letzten Jahren das Interesse an Fragen der Temporalität, wobei sowohl kurz- als auch längerfristige physische Interaktionskonstellationen in den Fokus rückten. Trotz der starken empirischen wie theoretischen Ausdifferenzierung physischer Kopräsenzen wurden zwei Aspekte bisher kaum ausreichend problematisiert: erstens das dichotome Verständnis physischer Kopräsenz (das sich auf die binäre Differenzierung von Abwesenheit und Anwesenheit reduziert) und zweitens die Konzeption virtueller Kopräsenz als eine defizitäre Version von Kommunikation (der das breite Spektrum sinnlicher Dimensionen von Faceto-face-Interaktionen fehlt). Zur Problematisierung dieser Aspekte von Kopräsenz reichern wir unsere geographische Argumentation mit Befunden aus benachbarten Disziplinen an und fokussieren die durch die Virtualisierung implizierten Veränderungen der Kommunikationskontexte. Hierbei stellen insbesondere die komplexen Verflechtungen von Offline- und Online-Interaktionen die etablierten geographischen Zugänge vor methodische und konzeptionelle Herausforderungen. Zur Bewältigung dieser Herausforderungen wird eine Erweiterung des zeitgeographischen Modells von Torsten Hägerstrand um eine virtuelle Dimension vorgeschlagen. Ziel unserer Erweiterung ist es, raumzeitliche Beschränkungen kollaborativer Prozesse sowohl in physischen als auch in virtuellen Kontexten sowie in (physisch-virtuellen) synthetischen Situationen empirisch erfassbar und vergleichbar zu machen. Unsere methodisch-konzeptionelle Erweiterung der Zeitgeographie illustrieren wir mit empirischen Vignetten kollaborativer Prozesse aus den Feldern Kunst (Musikproduktion im Studio) und Wissenschaft (Pharmaforschung im Labor).

\section{Schlüsselwörter: Hägerstrand, Zeitgeographie, Virtualität, Kopräsenz, Labor, Studio}

\begin{abstract}
In economic geography, during the last years interest in various aspects of temporality increased, both in the context of short- as well as longer-term physical interaction constellations. Despite the empirical and theoretical differentiation of physical co-presence, two aspects have not been problematized in a sufficiently systematic fashion so far: first, the dichotomous understanding of physical co-presence (that is reduced to a binary differentiation of presence/absence) and, second, the conception of virtual co-presence as a deficient version of communication (that lacks the broad spectrum of sensual cues of face-to-face interaction). To overcome the insufficient conceptualization

\footnotetext{
*Corresponding author: Alice Melchior, Hafencity Universität Hamburg, Arbeitsgebiet Stadt- und Regionalökonomie, Überseeallee 16, 20457 Hamburg, Deutschland, E-mail: alice.melchior@hcu-hamburg.de, ORCID: 0000-0001-8441-2392

Benjamin Schiemer, Johannes-Kepler-Universität Linz, Institut für Organisation, Altenberger Straße 69, 4040 Linz, Österreich Prof. Dr. Gernot Grabher, Hafencity Universität Hamburg, Arbeitsgebiet Stadt- und Regionalökonomie, Überseeallee 16, 20457 Hamburg, Deutschland
} 
of co-presence we enrich our geographical argumentation with findings from adjacent disciplines and focus on the modification in communication contexts implied by virtualization. In particular, the intricate entanglement of offline and online interaction dynamics is challenging the established methodical and conceptual geographical approaches. In order to deal with these challenges, we seek to advance the time-geographic model of Torsten Hägerstrand by including the virtual dimension of interactions. The aim of our extension is the development of a methodical template that is suitable to conceptualize empirically space-time trajectories in physical, virtual and (physical-virtual) synthetic contexts. We illustrate our methodical-conceptual extension of the time-geographic model with empirical vignettes from the fields of art (music production in a studio) and science (pharmaceutical research in a laboratory).

Keywords: Hägerstrand, Time geography, Virtuality, Co-presence, Laboratory, Studio

\section{Einleitung}

Das Interesse der Wirtschaftsgeographie verschob sich in den letzten Jahren von permanenten physischen Interaktionskonstellationen, etwa in territorialen Innovationssystemen wie Industrial Districts oder Clustern (Bündeln), zunehmend hin zu temporären Kooperationen. Das steigende Interesse an der Frage der Temporalität erstreckt sich von sehr kurzfristigen physischen Konstellationen wie Konferenzen (Maskell/Bathelt/ Malmberg 2006) über mittelfristige Kooperationen im Rahmen von Projekten (Grabher 2002) bis hin zu längerfristigen physischen Interaktionskonstellationen wie etwa im Rahmen von Forschungsaufenthalten (Torre/ Rallet 2005). Mit der Fokusverschiebung erfolgte eine starke empirische und theoretische Ausdifferenzierung der temporären physischen Kopräsenz, wobei zwei Aspekte vor dem Hintergrund der zunehmenden Virtualisierung bisher nur unzureichend problematisiert wurden: erstens blieb der Begriff Kopräsenz lange auf ein dichotomes und physisches Verständnis von Kopräsenz beschränkt, das lediglich eine binäre Differenzierung von kopräsent/nichtkopräsent und keinerlei Mischformen (wie etwa kopräsent, aber nicht verfügbar) kennt; zweitens dominierte eine Konzeption von virtueller Kopräsenz als unvollständigem Substitut für physische Kopräsenz (Grabher/lbert 2014). Zur Problematisierung beider Annahmen reichern wir unsere geographische Argumentation mit relevanten Befunden aus benachbarten Disziplinen und Forschungsfeldern wie der Soziologie und der Wissenschafts- und Technikforschung an.

Die zunehmende Virtualisierung in allen Bereichen der Kommunikation ermöglicht zum einen Kopräsenzen zwischen physisch entfernten Akteuren (Grabher/ Maintz 2007; Campos-Castillo 2012) sowie Kopräsenzen zwischen Akteuren und technologischen Artefakten wie beispielsweise elektronisch übertragene On-screenProjektionen (Knorr Cetina 2009: 65 f.). Zum anderen sind virtuellen Kopräsenzen eigene Möglichkeiten und
Grenzen der Kommunikation inhärent. Die Asynchronität virtueller Kommunikationsprozesse bietet gegenüber Face-to-face-Interaktionen beispielsweise die Vorteile, Argumente mit Belegmaterial (wie etwa Internetlinks oder Abbildungen) zu untermauern, Modifikationen einer möglichen Lösung zu testen (Grabher/lbert 2014) sowie Gelegenheiten zum reflective reframing (Hargadon/Bechky 2006: 489 ff.) bis hin zur Umstrukturierung eigener Wissensbestände etwa durch spezifische Anfragen oder Anregungen anderer Interaktionspartner. Virtuelle Kopräsenz, so einer unserer zentralen Ausgangspunkte, lässt sich daher nicht allein auf eine defizitäre Version von physischer Kopräsenz reduzieren (Bathelt/ Turi 2011), sondern stellt vielmehr einen Kontext sui generis dar, der zum integrierten Bestandteil kollaborativer Prozesse geworden ist (Grabher/Melchior/Schiemer et al. 2018). Darauf aufbauend gehen wir davon aus, dass das Verständnis von Kopräsenz als "being there“ (das heißt, den gleichen physischen Raum mit anderen Akteuren zu teilen) im Zuge der Virtualisierung durch ein Verständnis von Kopräsenz als "being aware“ (das heißt sich aktiv gegenseitig aneinander orientieren) verstanden werden sollte (Grabher/Melchior/Schiemer et al. 2018).

Studien der Wissenschafts- und Technikforschung (z. B. Knorr Cetina 2009) sowie soziologische Auseinandersetzungen (z. B. Zhao 2003; Zhao/Elesh 2008; Campos-Castillo 2012; Houben 2018) demonstrieren darüber hinaus, dass virtuelle Kopräsenzen in ihrer geographischen und soziodemographischen Reichweite sowie in ihrer Informationstiefe dramatisch zugenommen haben. Die zunehmende Gleichzeitigkeit und Verschmelzung von Kopräsenz/Abwesenheit sowie von physischen/virtuellen Interaktionen führt vermehrt zu dem, was Knorr Cetina (2009: 61) als „synthetische Situationen" bezeichnet. Synthetische Situationen umfassen elektronisch übertragene On-screen-Projektionen, die dem Kommunikationskontext sowohl Informationstiefe wie auch neue Reaktionsanforderungen hinzufügen. 
In einer Video-Konferenz beispielsweise interagieren zwei oder mehr Akteure virtuell miteinander (response presence), während sich jeder Akteur in seiner eigenen physischen Umgebung befindet (embodied presence). Bedingt durch die vermehrte Nutzung von Informationsund Kommunikationstechnologien beschreibt die synthetische Situation eine neuartige und ubiquitäre Form der Kopräsenz, bei der sich die physische Umgebung (physische Kopräsenz) und die virtuelle On-screen-Projektion (virtuelle Kopräsenz) gegenseitig bedingen.

Das Verständnis von Kopräsenz als „being aware“ und die Omnipräsenz synthetischer Situationen stellt die etablierten geographischen Zugänge vor methodische und empirische Herausforderungen. Erstens muss der Fluss von Interaktionssequenzen mit einer Granularität erfasst werden, die es erlaubt, die Dynamiken der Kopräsenzen über mehrere Kommunikationskontexte hinweg (z. B. Diskussion eines Experiments in einer SkypeKonferenz, Durchführung des Experiments im Labor, Zirkulation der Ergebnisse per E-Mail, Arbeitskoordination über Beiträge (postings) in geschlossenen Facebook-Gruppen) durch prozessorientierte und standortübergreifende Forschung zu verfolgen und sinnvoll zu interpretieren. Zweitens müssen hierbei nicht nur die raumzeitlichen Beschränkungen physischer Kopräsenzen, sondern auch die Chancen und Grenzen virtueller Kopräsenzen berücksichtigt werden, wobei vor allem die Simultanität von physischer und virtueller Kopräsenz in synthetischen Situationen (z. B. Skype-Konferenz) eine konzeptionelle wie methodische Herausforderung darstellt (Grabher/Melchior/Schiemer et al. 2018). Zur Bewältigung dieser Herausforderungen entwickeln wir im Folgenden ein methodisches Instrument zur Erfassung und zum Vergleich raumzeitlicher Aktivitätsmuster innerhalb kollaborativer Prozesse. Als tragfähiger Ausgangspunkt wurde das zeitgeographische Konzept von Hägerstrand gewählt, da es mit seinem Fokus auf die raumzeitlichen Bedingungen des individuellen Handelns die Konzeptualisierung und empirische Erfassung von Raum-Zeit-Trajektorien in physischen Kontexten ermöglicht. In Anlehnung an die bisherigen Erweiterungen der klassischen Zeitgeographie (z. B. Kwan 2000; Kwan 2004; Miller 2005; Schwanen/Kwan 2008; Yu/ Shaw 2008; Shaw/Yu 2009; Farber/Neutens/Miller et al. 2013; Miller 2017) versuchen wir aus einer qualitativen Perspektive heraus, die Modifikationen und vor allem die Generierung neuer Beschränkungen kollaborativer (Arbeits)praktiken systematisch zu erfassen. In Abgrenzung zu den bisherigen quantitativen Ansätzen versuchen wir nicht, die klassische Zeitgeographie für GISbasierte Daten fruchtbar zu machen, sondern entwickeln eine methodisch-konzeptionelle Erweiterung der klassischen Zeitgeographie um eine virtuelle Dimension.

Zur Entwicklung unserer konzeptionell-methodischen Erweiterung werden wir zuerst die grundlegenden Begriffe und theoretischen Ausgangspunkte der Zeitgeographie erläutern sowie die grundlegende Kritik am Modell der klassischen Zeitgeographie vorstellen (Kapitel 2.1). In einem folgenden Schritt werden wir in Anlehnung an bisherige Erweiterungen der Zeitgeographie die Modifikationen der raumzeitlichen Beschränkungen durch virtuelle Kopräsenzen sowie die Generierung neuer Beschränkungen systematisch analysieren, um anschließend eine konzeptionell-methodische Erweiterung der Zeitgeographie zu erarbeiten (Kapitel 2.2). Anknüpfend illustrieren wir unsere vorgeschlagene Erweiterung mit ethnographischen Vignetten aus unserer empirischen Erforschung kollaborativer Prozesse in den Feldern Kunst (Musikproduktion im Studio) und Wissenschaft (pharmazeutische Forschung im Labor) (Kapitel 3). ${ }^{1}$ In Kapitel 4 werden die Ergebnisse zusammengefasst.

\section{Konzeptionell-methodische Erweiterung des zeitgeographi- schen Modells}

\subsection{Theoretische Ausgangspunkte und begriffliche Grundlagen der Zeitgeographie}

Theoretische Prämisse der Zeitgeographie ist die Untrennbarkeit von Raum und Zeit: Jeder Akteur folgt einem individuellen Pfad durch ein Raum-Zeit-Kontinuum (Hägerstrand 1970: 9 f.). Diese individuellen Pfade sind durch das Zusammenspiel unterschiedlicher raumzeitlicher Beschränkungen („capability, coupling and authority constraints") konditioniert (Hägerstrand 1970: $10 \mathrm{ff}$.). Auf der Grundlage der biologischen Konstruktion des menschlichen Körpers wird der Handlungsspielraum jedes Akteurs durch die Kapazitätsbeschränkungen begrenzt: Akteure können sich physisch grundsätzlich nur an einem Ort aufhalten. Die Kopplungsbeschränkungen hingegen begrenzen den Ort, den Zeitpunkt und

1 Die vorgestellten empirischen Befunde wurden im Kontext der interdisziplinären Forschergruppe „Organized Creativity. Practices for Inducing and Coping with Uncertainty" der Deutschen Forschungsgemeinschaft (DFG FOR 2161) erhoben. 
die Dauer für Kopräsenzen (Hägerstrand 1970: 14 ff.), wodurch die Bündelung von Akteuren (etwa in Besprechungen) sowie von Akteuren und Objekten (etwa Arbeiten am Mikroskop) ermöglicht und beschränkt wird (Thrift 1977: 7). Als drittes limitieren die Autoritätsbeschränkungen (wie etwa Zugangskriterien oder Öffnungszeiten) den Zugang zu konkreten Lokalitäten, innerhalb derer Bündel organisiert werden (Thrift 1977: 7). Aufbauend auf der Annahme der Untrennbarkeit von Raum und Zeit bedingen sich alle drei Beschränkungen wechselseitig auf direkte oder indirekte Art (Hägerstrand 1970: 17). Trotzt der Berücksichtigung des Umstandes, dass sich mit dem Aufkommen der Kommunikations- und Informationstechnologien die Interdependenzen der raumzeitlichen Beschränkungen auf grundlegende Weise verändern (Hägerstrand 1970: 15 ff.), beschränkt sich das Konzept der Zeitgeographie primär auf synchrone physische Kopräsenzen wie persönliche Gespräche, bei denen die beteiligten Akteure physisch anwesend sind (Miller 2017). Asynchrone physische Kopräsenzen wie etwa Notizen an einer Bürotür oder Eintragungen im Laborbuch werden zwar von Hägerstrand (1970) berücksichtigt, aber nicht systematisch in die Zeitgeographie integriert (Miller 2017). Ähnlich wie asynchrone physische Kopräsenzen wird die Möglichkeit der virtuellen Kopräsenz von Hägerstrand in Form eines Telefongesprächs (synchrone virtuelle Kopräsenz) zwar angesprochen, jedoch nicht konsequent weiterentwickelt (Miller 2017). Des Weiteren werden asynchrone virtuelle Kopräsenzformen wie beispielsweise E-Mails oder Webseiten genau wie synchrone virtuelle Kopräsenzen theoretisch generell einbezogen, doch in der klassischen Zeitgeographie ignoriert (Miller 2017).

Es existiert eine Vielzahl quantitativ angelegter Erweiterungsansätze, welche die Grenzen der klassischen Zeitgeographie aufzeigen und vorzugsweise den Einfluss virtueller Kopräsenzen auf Aktivitäts- und Reisemuster von Akteuren erfassen. So konnte die Studie von Shaw und Yu (2009) zeigen, dass sich sowohl die Kapazitätsbeschränkungen als auch die Kopplungsbeschränkungen von Reisenden durch die neuen Kommunikations- und Informationstechnologien verändern. Die Kapazitäts- und Kopplungsbeschränkungen werden insoweit reduziert, als dass die Akteure von der Notwendigkeit der physischen Präsenz an einem konkreten Ort befreit werden und daher an mehr als einem Bündel gleichzeitig teilnehmen können (Shaw/Yu 2009). Beispielsweise können Akteure mit einem physisch dispersen Akteur per Telefon diskutieren, gleichzeitig eine E-Mail an einen anderen Akteur verfassen und simultan auf einer Website nach weiteren Informationen für die
Diskussion am Telefon suchen. In Hinblick auf die Autoritätsbeschränkungen ermöglichen virtuelle Kopräsenzen beispielsweise das Umgehen von Ladenöffnungszeiten (Schwanen/Kwan 2008) sowie die Perforation von rigiden Arbeitszeitregimen durch Home-Office-Arbeitsplätze. Die bisherigen Untersuchungen legen nahe, dass die Kommunikations- und Informationstechnologien die raumzeitlichen Beschränkungen im Alltag grundlegend verändern (Schwanen/Kwan 2008), indem sie diese nicht nur relativieren, sondern durch ihre ganz eigenen Rhythmen und Ordnungen neue Einschränkungen für das tägliche Leben generieren (Shaw/Yu 2009). Die Ubiquität synthetischer Situationen, in denen Onscreen-Projektion neue Informationstiefen und Reaktionsanforderungen generieren (Knorr Cetina 2009: 61), ist die Folge.

Wie sich die Veränderungen der raumzeitlichen Beschränkungen im Zuge der Virtualisierung in kollaborativen Arbeitsprozessen auswirken, wird im nächsten Schritt systematisch analysiert. Im Fokus der Analyse steht nicht nur die Relativierung der Beschränkungen, sondern vielmehr die Generierung neuer raumzeitlicher Beschränkungen, die nicht nur durch neue Informations- und Kommunikationstechnologien, sondern auch im Zusammenspiel mit der Virtualisierung von Objekten (etwa die Digitalisierung von Musik oder die partielle Digitalisierung von Zellen), die einen teilweise friktionsIosen Austausch in kollaborativen Kontexten ermöglicht, entstehen.

\subsection{Ein methodisches Konzept zur empi- rischen Analyse von Raum-Zeit-Trajekto- rien in kollaborativen Prozessen}

Generell besteht ein breiter Konsens darüber, dass die Kommunikations- und Informationstechnologien raumzeitliche Beschränkungen aufheben (Schwanen/Kwan 2008). In welchem Ausmaß diese Relativierungen auftreten wird hingegen konträr diskutiert. Beispielsweise argumentiert Couclelis (2000; 2004), dass die Assoziation von Aktivität, Ort und Zeit (raumzeitlich fixierte Aktivität) durch virtuelle Kopräsenzen geschwächt wird und dass Aktivität zunehmend über verschiedene Zeiten und geographische Standorte verteilt (raumzeitlich flexible Aktivität) stattfindet. Andere Forscher (z. B. Kwan 2001; Dijst 2004; Zook/Dodge/Aoyama et al. 2004) hingegen relativieren diese Nivellierung der raumzeitlichen Beschränkungen mit der Argumentation, dass OnlineAktivitäten durch den Akteur immer im physischen Raum verankert sind und dass virtuelle Räume dementspre- 
chend in soziale und physische Räumen eingebettet sind. Die mehrdeutigen und paradoxen Auswirkungen der Kommunikations- und Informationstechnologien auf die raumzeitlichen Beschränkungen sind demnach von Technologien und anderen beteiligten Materialien sowie ihrem Kontext beeinflusst (Schwanen/Kwan 2008). Ziel unserer methodisch-konzeptionellen Erweiterung der Zeitgeographie ist es daher, die drei klassischen Formen von raumzeitlichen Beschränkungen im Lichte virtueller Interaktionsmodi hinsichtlich ihrer Auswirkungen auf kollaborative (Arbeits-) Praktiken genauer zu betrachten. Hierzu werden zuerst die Veränderungen der Kapazitäts-, Kopplungs- und Autoritätsbeschränkungen für kollaborative Praktiken diskutiert, um anschließend systematisch auf die Generierung neuer Beschränkungen einzugehen.

Das Aufkommen der Kommunikations- und Informationstechnologien wie Mobiltelefon, E-Mail oder Skype hat die Kapazitätsbeschränkungen auf nachhaltige und durchaus ambivalente Weise verändert (vgl. Tabelle 1). Einerseits wurden Akteure durch die Möglichkeiten virtueller Interaktion vom Zwang der physischen Präsenz an einem konkreten Ort befreit, andererseits generieren virtuelle Kopräsenzen neue Kapazitätsbeschränkungen.

Die vermehrte Nutzung virtueller Interaktionsmodi führt zunehmend zur Gleichzeitigkeit von Online- und Offline-Kontexten, wobei diese mit unterschiedlichen Einschränkungen hinsichtlich der Aufmerksamkeit, Autorität, sozialen Erwartungen und Dauer verbunden sind (Thulin/Vilhelmson 2018: 97 f.). E-Mails sollten beispielsweise spätestens nach einer kulturell normierten Zeitdauer (ohne unangemessene Verzögerung) beantwortet werden, was einer (expliziten oder impliziten) Erwiderungsverantwortung entspricht. Die neu generierte Erwiderungsverantwortung stellt somit eine neue Kapazitätsbeschränkung dar, die als „response presence“ (Knorr Cetina 2009: 69 ff.) bezeichnet werden kann. Darüber hinaus generieren virtuelle Kopräsenzen mit ihrer response presence einen neuen Modus der körperlichen Affektivität, der eine ständige Überwachung und sensorische Abstimmung der Informations- und Kommunikationstechnologien (wie etwa den reflexhaften Blick auf den Handy-Bildschirm) einfordert (Knorr Cetina 2009: 75).

Hinsichtlich der Kopplungsbeschränkungen führen neue Kommunikations- und Informationstechnologien zu einer Veränderung der Koordinierung von Akteuren, sodass beispielsweise bevorstehende Verspätungen direkt kommuniziert werden können, was zur Flexibilisierung raumzeitlich festgelegter Tätigkeiten führt (Schwanen/Kwan 2008). Die Zunahme interaktionsbasierter
Koordinierungsstile als Ergänzung oder Ersatz der "clock-based“2 (Ling 2004: 58) Koordinierung führt zur Verschiebung ortsbezogener Konnektivität hin zur individuellen Mensch-zu-Mensch-Konnektivität (Wellman 2001; Kwan 2007). Zusätzlich wird raumzeitlich fixierte Aktivität zumindest teilweise für Flexibilität und die Möglichkeit, Ressourcen so effizient wie möglich neu zuzuordnen, eingetauscht (Townsend 2000). Die partielle Suspendierung der Imperative des gemeinsamen Ortes, des abgestimmten Zeitpunktes sowie der vereinbarten Dauer zur Bündelung von Akteuren und Objekten (von raumzeitlich fixierte Aktivität hin zu raumzeitlich flexibler Aktivität) generiert jedoch neue raumzeitliche Kopplungsbeschränkungen (vgl. Tabelle 1). Erstens müssen die materiellen Vorrichtungen für virtuelle Kopräsenzen im physischen Raum platziert werden (Schwanen/Kwan 2008): Beispielsweise werden internetfähige Computer (materielle Vorrichtung) für das Beantworten von E-Mails (virtuelle Kopräsenz) im Büro (physischer Raum) benötigt. Zweitens müssen die materiellen Vorrichtungen für die virtuelle Kopräsenz mit den Akteuren, die sie nutzen wollen gebündelt werden (Pred 1978; Kwan 2001; Schwanen 2007). Diese Beschränkungen gelten sowohl für kabelgebundene als auch für mobile Kommunikations- und Informationstechnologien. Darüber hinaus müssen an den materiellen Vorrichtungen Wartungsarbeiten zur Aufrechterhaltung der Möglichkeit zur virtuellen Kopräsenz durchgeführt werden, wie etwa das Mitführen von Geräten oder das Aufladen von Batterien und Akkus (Ling 2004).

Neben neu entstehenden Kommunikationsmöglichkeiten veränderten sich im Zuge der Virtualisierung auch die Objekte kollaborativer Arbeitsprozesse (vgl. Tabelle 1). Die generelle Möglichkeit der Virtualisierung von Objekten trägt zur Entkopplung zwischen Objekt und Akteur bei, indem virtualisierte Objekte über große Distanzen hinweg zirkuliert und in synchroner oder asynchroner Kopräsenz bearbeitet werden können. Akteure müssen beispielsweise nicht mehr ihren physischen Büroarbeitsplatz aufsuchen, sondern können raumzeitlich entkoppelt von ihrem Laptop aus an virtualisierten Objekten arbeiten.

Die veränderten Kapazitäts- und Kopplungsbeschränkungen tragen dazu bei, dass sich auch die Autoritätsbeschränkungen verändern (vgl. Tabelle 1). Die Virtualisierung von Objekten sowie virtuelle Interaktions-

2 Mit der "clock-based“ (Ling 2004: 58) Koordinierung ist die taktbasierte Aktivitätsplanung auf Basis eines gemeinsamen Messsystems - der Zeit (Uhrzeit) - gemeint (vgl. Schwanen/Kwan 2008). 
Tabelle 1: Veränderungen der raumzeitlichen Beschränkungen durch die Virtualisierung

\begin{tabular}{llll}
\hline & $\begin{array}{l}\text { Reduzierung durch } \\
\text { Virtualisierung }\end{array}$ & $\begin{array}{l}\text { Extension durch } \\
\text { Virtualisierung }\end{array}$ & $\begin{array}{l}\text { Neue Beschränkungen durch } \\
\text { Virtualisierung }\end{array}$ \\
\hline $\begin{array}{l}\text { Kapazitäts- } \\
\text { beschränkungen }\end{array}$ & $\begin{array}{l}\text { Notwendigkeit der physischen } \\
\text { Anwesenheit }\end{array}$ & $\begin{array}{l}\text { Virtuelle Kopräsenzen und } \\
\text { synthetische Situationen }\end{array}$ & $\begin{array}{l}\text { Response presence und permanente } \\
\text { körperliche Affektivität }\end{array}$ \\
$\begin{array}{l}\text { Kopplungs- } \\
\text { beschränkungen }\end{array}$ & $\begin{array}{l}\text { Raumzeitliche Kopplung von } \\
\text { Akteur und Objekt (raumzeitlich } \\
\text { fixierte Aktivität) }\end{array}$ & $\begin{array}{l}\text { Virtualisierung von Objekten } \\
\text { und Bündeln (raumzeitlich } \\
\text { flexible Aktivität) }\end{array}$ & $\begin{array}{l}\text { Materielle Vorrichtungen für virtuelle } \\
\text { Kopräsenzen müssen im physischen }\end{array}$ \\
& & & $\begin{array}{l}\text { Raum verankert, mit Akteuren koordiniert } \\
\text { und gewartet werden. }\end{array}$ \\
Autoritäts- & Physische Zugangskontrollen \\
beschränkungen & Virtuelle Bündel mit virtuellen & Virtuelle Zugangskontrollen, Sicherheits- \\
& & Zugangskontrollen & und Schutzmaßnahmen sowie Grad der \\
& & & Virtualisierung des Objektes \\
\hline
\end{tabular}

medien wie beispielsweise Online-Foren, Chat-Gruppen, Facebook-Gruppen, E-Mail-Verteiler und Gruppen in Instant-Messaging-Diensten wie WhatsApp und Slack ${ }^{3}$ erschließen fundamental neue Formen der Kopräsenz und Kollaboration. Wurde der Zugang zu physischen Lokalitäten (und ihren Bündeln) traditionell noch durch physische Zugangskontrollen wie Pförtner, Türen oder Schlösser kontrolliert, sind es vor allem virtuelle Mitgliedschaften, die den Zugang zu virtuellen Bündeln kontrollieren und beschränken. Darüber hinaus entstehen neue Autoritätsbeschränkungen, die die Nutzung von Kommunikations- und Informationstechnologien limitieren (Schwanen/Kwan 2008). Als Reaktion auf die vermehrten virtuellen Kopräsenzen wurde beispielsweise die Nutzung von mobilen Endgeräten bei Start und Landung von Flugzeugen verboten und es wird vermehrt darum gebeten, Mobiltelefone in Theatern, Kinos und einigen Restaurants (Harvey/Macnab 2000; Dijst 2004) aus Lärmschutzgründen aus- oder zumindest lautlos zu stellen. Zusätzlich zum Zugang zu virtuellen Bündeln, reguliert durch virtuelle Mitgliedschaften oder neukonstruierte Sicherheits- und Schutzmaßnahmen, stellt auch der Grad der Virtualisierung des Objektes eine neue und objektbezogene Autoritätsbeschränkung des kollaborativen Arbeitsprozesses dar, da virtualisierte Objekte einen anderen raumzeitlichen Zugang ermöglichen als nichtvirtualisierte Objekte.

\section{Empirische Anwendung des Konzeptes}

Zur Illustration der methodisch-konzeptionellen Erweiterung der Zeitgeographie stellen wir im Folgenden zwei Vignetten unserer empirischen Forschung zu kollaborativen Prozessen in den Feldern Kunst (Musikproduktion im Studio) und Wissenschaft (Pharmaforschung im Labor) vor.

\subsection{Forschungsdesign, Fallauswahl und Datenerhebung}

Labor und Studio wurden für die komparative Analyse der Raum-Zeit-Trajektorien aus den Feldern der Kunst und der Wissenschaft ausgewählt, da sowohl in der Musikindustrie als auch in der Pharmaforschung kollaborative Prozesse immer einen konkreten physischen Aufnahme-Kontext (Studio) oder einen wissenschaftlichen Test-Kontext (Labor) durchlaufen. Beiden Kontexten ist gemein, dass mehrere Akteure am Prozess beteiligt sind und dass die raumzeitliche Koordinierung von Akteuren und Objekten innerhalb des Prozesses eine komplexe Herausforderung darstellt. Gleichwohl unterscheiden sich beide Kontexte in Hinblick auf die Virtualisierung der zentralen Arbeitsobjekte: Während im Studio der Sound virtualisiert, digital bearbeitet und beinahe beliebig modelliert werden kann, steht diese Option bei Zellen im Labor nur partiell zur Verfügung.

Um die Raum-Zeit Trajektorien in beiden Kontexten (Labor und Studio) zu erfassen, wurde eine ethnographische $^{4}$ Erhebungsstrategie mit teilnehmenden Beobach-

4 Für detaillierte Informationen zur Durchführung von

Ethnographien vgl. beispielsweise Creswell (2007).
3 Slack ist ein webbasierter Instant-Messaging-Dienst, der es erlaubt, innerhalb von Gruppen Nachrichten auszutauschen, zu chatten sowie an gemeinsamen Dokumenten zu arbeiten. 
Tabelle 2: Übersicht der teilnehmenden Beobachtungen in Labor und Studio

\begin{tabular}{|c|c|c|c|c|c|}
\hline & Art & Dauer & Erhebungsorte & Datentypen & Auswertung \\
\hline \multirow[t]{3}{*}{ Labor } & $\begin{array}{l}\text { Teilnehmende } \\
\text { Beobachtungen im Labor }\end{array}$ & $40 \mathrm{~h}$ & $\begin{array}{l}\text { Großraumbüro, } \\
\text { Laboratorien, } \\
\text { Mikroskop-Raum }\end{array}$ & Feldnotizen, Fotos & Inhaltsanalyse \\
\hline & $\begin{array}{l}\text { Teilnehmende } \\
\text { Beobachtungen von } \\
\text { Meetings }\end{array}$ & $19 \mathrm{~h}$ & Konferenzraum & Interaktionsprotokolle, Feldnotizen & \\
\hline & $\begin{array}{l}\text { Teilnehmende } \\
\text { Beobachtungen } \\
\text { (shadowing) von Akteuren }\end{array}$ & $43 \mathrm{~h}$ & $\begin{array}{l}\text { Orte, die der Akteur } \\
\text { aufsuchte }\end{array}$ & $\begin{array}{l}\text { Bewegungs- und Interaktionsprotokolle, } \\
\text { Feldnotizen }\end{array}$ & \\
\hline \multirow[t]{2}{*}{ Studio } & $\begin{array}{l}\text { Teilnehmende } \\
\text { Beobachtungen im Studio }\end{array}$ & $120 \mathrm{~h}$ & Studio & $\begin{array}{l}\text { Feldnotizen, Interaktions- und } \\
\text { Bewegungsprotokolle, Audioaufnahmen } \\
\text { von Interaktionen, Fotos }\end{array}$ & Inhaltsanalyse \\
\hline & $\begin{array}{l}\text { Beobachtungen der } \\
\text { Online-Interaktionen auf } \\
\text { Facebook und Slack }\end{array}$ & $40 \mathrm{~h}$ & $\begin{array}{l}\text { Geschlossene } \\
\text { Facebook-Gruppe, } \\
\text { geschlossene } \\
\text { Slack-Gruppe }\end{array}$ & Bildschirmfotos (screenshots), Feldnotizen & \\
\hline
\end{tabular}

tungen gewählt. Die teilnehmende Beobachtung ist eine Methode der Ethnographie (vgl. Geertz 1973), bestehend aus der physischen Präsenz am Erhebungsort, der Teilnahme am Arbeitsalltag und einer detailreichen Aufzeichnung von Interaktionen, informellen Interviews und Beobachtungen von Alltags- und Arbeitsabläufen (vgl. Girtler 2001). Während des jeweils zweiwöchigen Beobachtungszeitraumes wurden im Labor sowohl physische Orte (Großraumbüro, Laboratorien und der Mikroskop-Raum) und ihre darin stattfinden (physischen und virtuellen) Kopräsenzen als auch einzelne Akteure teilnehmend begleitet (shadowing) und beobachtet (vgl. Tabelle 2). Im Studio hingegen war für die teilnehmende Beobachtung der Akteure ein Shadowing nicht notwendig, da die aufgesuchten physischen Orte von einem Punkt aus beobachtbar waren. Zusätzlich wurden im Studio Online-Interaktionen auf Facebook und Slack netnographisch $^{5}$ (vgl. Kozinets 2009) erhoben (vgl. Tabelle 2), da diese besonders häufig für virtuelle Kopräsenzen genutzt wurden. Die beobachteten Raum-Zeit-Trajektorien wurden jeweils mithilfe von Feldnotizen, Fotos, Interaktions- und Bewegungsprotokollen festgehalten. Anschließend wurden die empirischen Daten inhaltsanalytisch (vgl. Mayring 2008) ausgewertet.

5 Netnographie ist eine Forschungsmethodik, welche an die Ethnographie angelehnt ist und zur Beobachtung, Erhebung und Erfassung von Online-Interaktionen und der Nutzung von virtuellen Medien dient (vgl. Kozinets 2009).

\subsection{Die Ausgangssituationen: Labor und Studio}

Das untersuchte pharmazeutische Labor ist auf einem Forschungscampus in der Umgebung von Berlin angesiedelt. Die Räumlichkeiten der beobachteten Laborgruppe liegen im dritten Stockwerk eines Forschungsgebäudes und beheimaten vier unterschiedliche Laboratorien mit Laborgeräten, Kühlschränken und Brutschränken, in denen die benötigten Zellen, Viren und Tiere gehalten werden, sowie einem Mikroskop-Raum, mehrere Büroräume mit individuellen Computerarbeitsplätzen, einen Konferenzraum, zwei Lagerräume, eine Küche und ein WC. Während des zweiwöchigen Beobachtungszeitraumes waren wochentags meist alle 20 wissenschaftlichen Mitarbeiterinnen/Mitarbeiter der Forschungsgruppe auf dem Forschungscampus physisch anwesend. In mehreren kleineren Teams arbeiteten sie in verschiedenen Lokalitäten sowohl an individuellen Dissertationsprojekten sowie an langjährigen kollaborativen Projekten. Vormittags wurden überwiegend individuelle Laborarbeiten durchgeführt, nachmittags fanden vor allem Meetings der einzelnen Teams und Ad-hoc-Besprechungen zur Diskussion des aktuellen Arbeitsstandes im Großraumbüro statt. Am Wochenende wurde vereinzelt im Labor gearbeitet, wenn der zelluläre Prozess eine ununterbrochene Observation erforderte. Zur längerfristigen Koordination der einzelnen Teams und der gesamten Forschungsgruppe wurden vor allem E-Mail-Verteil-Listen, Doodle-Umfragen und gemeinsame virtuelle Kalender 
genutzt sowie WhatsApp-Gruppen zur spontanen Koordination von einigen Teams.

Das untersuchte Tonstudio hat seinen Standort in Wien und wird von einem österreichischen Produzenten betrieben. Es liegt in den Kellerräumlichkeiten unter der Wohnung des Produzenten und verfügt über einen schalldichten Aufnahmeraum, einen Regieraum mit Hauptcomputer, einen Gemeinschaftsraum mit diversen Arbeitsplätzen und Nebencomputern, eine Küche sowie ein WC und ein Abstelllager. Während des zweiwöchigen Beobachtungszeitraumes arbeitete eine fünfköpfige Pop-Band in den Studioräumlichkeiten an der Aufnahme ihres Debütalbums. Der gesamte Aufnahmeprozess der Band vollzog sich in zwei sechswöchigen Arbeitsblöcken mit einer Pause von vier Monaten dazwischen. Der erste Block diente der Sammlung des künstlerischen Materials und für drei erste Aufnahmen. Im zweiten Block wurden aus dem von der Band in der Zwischenzeit weiterentwickelten Material sechs weitere Songs produziert. Zum Zeitpunkt der Beobachtungen befand sich die Band in der zweiten Woche des zweiten Blocks. Die erste Woche diente hauptsächlich zu Planungsgesprächen und der (Wieder-)Einrichtung des Studios. In den zwei Wochen der Beobachtungen befanden sich alle fünf Bandmitglieder, der Produzent und ein technischer Assistent beinahe ununterbrochen von 11:00 Uhr bis 23:00 Uhr im Studio. Es wurde arbeitsteilig an mehreren Arbeitsplätzen parallel gearbeitet. Der Produzent war mit Schlagzeug- und Bassaufnahmen beschäftigt, während sich die anderen Akteure auf die Weiterentwicklung einzelner Song-Arrangements konzentrierten. Parallel zur physischen Anwesenheit im Studio kommunizierten alle Akteure über eine geschlossene Facebook-Gruppe sowie über diverse andere virtuelle Kanäle wie Dropbox und dem Instant-Messaging-Dienst Slack.

\subsection{Raum-Zeit-Trajektorien in Labor und Studio}

Abbildung 1 zeigt eine typische Raum-Zeit-Trajektorie zweier Akteure während ihres kollaborativen Arbeitsprozesses im Labor, wobei ihre Handlungen durch die drei raumzeitlichen Beschränkungen ermöglicht und limitiert werden.

Zu Beginn des hier dargestellten Arbeitstages koordinieren sich die beiden wissenschaftlichen Mitarbeiter $A$ und $B$ zuerst in physischer Kopräsenz und später per Festnetztelefon (synchrone virtuelle Kopräsenz, reduzierte Kapazitätsbeschränkung). Um sich einen Einblick in den bisherigen Experimentverlauf zu verschaffen,

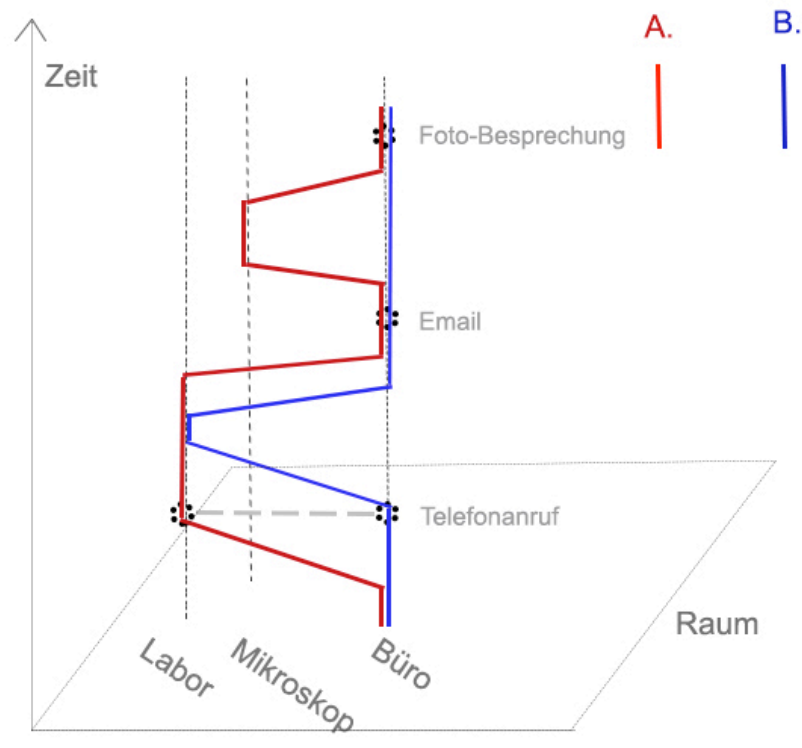

Abbildung 1: Bewegungsverhalten von zwei zusammenarbeitenden Akteuren im Labor

sucht Mitarbeiter B im Laufe des Vormittags seinen Kollegen A im Labor auf. In physischer Kopräsenz betrachten beide abwechselnd die Zellproben (Objekt) durch ein Mikroskop und diskutieren das Gesehene. Das Aufsuchen des Labors wird notwendig, da die Zellen nicht virtualisiert werden können und eine Bündelung von Akteur und Objekt (Zellen) nur in physischer Kopräsenz möglich ist. Anschließend verlässt $B$ das Labor wieder. Bevor auch $A$ das Labor verlässt, um seine E-Mails abzurufen und einen Blick auf sein Handy zu werfen, präpariert er Zellen für das am nächsten Tag geplante Experiment (Kopplungsbeschränkung). In dieser Sequenz wird erkennbar, dass die neuen Autoritätsbeschränkungen in Form von Schutz- und Sicherheitsvorkehrungen den Zugang zu virtuellen Bündeln stark limitieren. Zwar existiert im Labor ein Festnetztelefon, doch andere materielle Vorrichtungen für weitere virtuelle Kommunikationsmodi, wie beispielsweise ein internetfähiger Computer, sind im Labor nicht vorhanden. Auch das Mitführen eines Handys ist zwar nicht explizit verboten, doch aufgrund von genverändernden Arbeitssubstanzen und dem Vermeiden von Störgeräuschen aus Eigeninteresse unterlassen worden. Um Zugang zu seinen E-Mails (virtuelles Bündel) zu erlangen, sucht Mitarbeiter A das Großraumbüro auf, in dem in Form seines Arbeitscomputers die materielle Vorrichtung zur virtuellen Kopräsenz (in diesem Falle zum Abrufen von E-Mails) verankert ist (Kopplungs- und Autoritätsbeschränkung). Nach dem Abrufen und Beantworten einiger E-Mails verlässt $A$ das 
Büro wieder, um an einem speziellen Mikroskop im Mikroskop-Raum die Ergebnisse des am Vormittag durchgeführten Experimentes zu analysieren (Kopplungsbeschränkung). Um die Ergebnisse des Experimentes raumzeitlich entkoppelt mit seinem Kollegen B zu einem späteren Zeitpunkt im Großraumbüro sowie mit physisch dispersen Kollegen zu diskutieren, fotografiert A mithilfe des an das Mikroskop angeschlossenen Computers verschiedene Zellausschnitte seiner am Vormittag hergestellten Zellproben.

Während des zweiwöchigen Beobachtungszeitraumes waren die Akteure durch das bisher nicht virtualisierbare Objekt (lebende Zellen) physisch stark an das Objekt gekoppelt und mussten sowohl spezifische Räumlichkeiten (Labor, Mikroskop-Raum) als auch vom Objekt vorgegebene Zeitintervalle (Präparierung der Zellen) innerhalb ihres kollaborativen Prozesses einhalten, was die Vielzahl der raumzeitlich fixierten Tätigkeiten mit einem Clock-based-Koordinierungsstil erklärt. Um dieser Kopplung entgegenzuwirken, virtualisierten die Akteure die Ergebnisse ihrer Experimente, was insbesondere zu einer partiellen Entkopplung der Auswertung und Interpretation führte. Zur gemeinsamen Kommunikation nutzten die Akteure im Labor sowohl physische als auch virtuelle Kopräsenzen, wobei synthetische Situationen meist durch Echtzeitübertragungen von Mikroskopausschnitten oder das gemeinsame Interpretieren der virtualisierten Ergebnisse am Bildschirm innerhalb des kollaborativen Prozesses auftraten. Insgesamt wurde die partielle Entkopplung in Form einer Virtualisierung der Ergebnisse vor allem in Kombination mit der virtuellen (asynchronen) Kommunikation per E-Mail genutzt, um Ergebnisse gemeinsam mit physisch dispersen Akteuren zu teilen und zu diskutieren. Zusätzlich konnte festgestellt werden, dass die neuen Autoritätsbeschränkungen in Form von Sicherheits- und Schutzmaßnahmen in den Laboren (wie die Vermeidung von Störgeräuschen oder keine ans Internet angeschlossene Computer) dazu führten, dass die Akteure regelmäßig und aktiv das Großraumbüro aufsuchten, um dort Zugang zu entsprechenden virtuellen Kopräsenzen zu erlangen. Neben der häufig aktiv generierten physischen Kopräsenz nutzten die Akteure vor allem asynchrone virtuelle Kommunikationsmodi (mit Ausnahme des Festnetztelefons), da eine ununterbrochene Teilnahme an den virtuellen Bündeln durch die Schutz- und Sicherheitsvorkehrungen im Labor nicht möglich war.

Abbildung 2 zeigt eine typische Raum-Zeit-Trajektorie zweier Akteure während ihres kollaborativen Arbeitsprozesses im Studio. Wie im Labor werden ihre Hand-

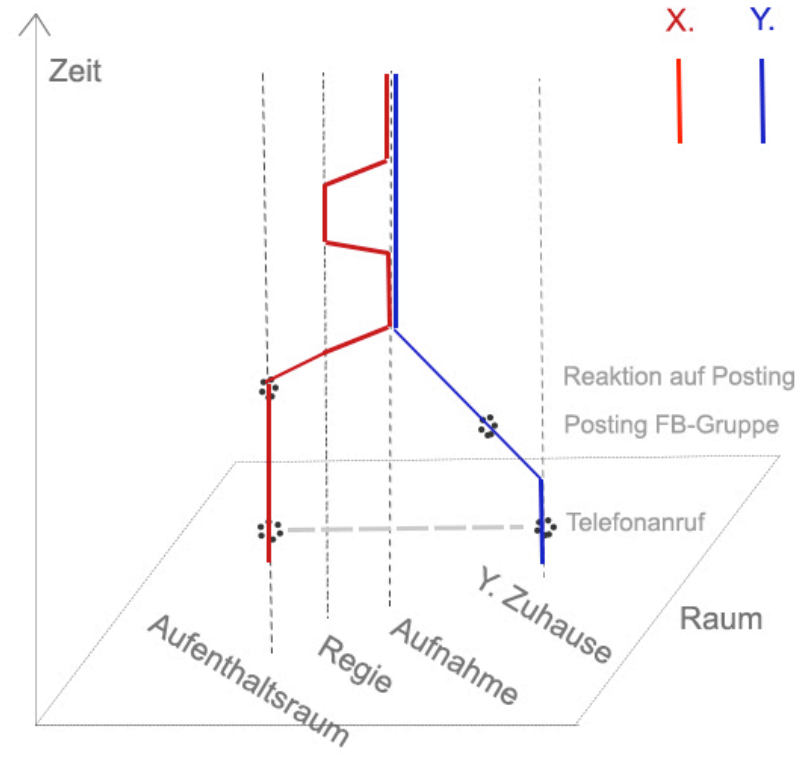

Abbildung 2: Bewegungsverhalten von zwei zusammenarbeitenden Akteuren im Tonstudio

lungen durch die drei raumzeitlichen Beschränkungen ermöglicht und limitiert.

Im Verlauf dieses Arbeitstages koordinieren Produzent $X$ und Schlagzeuger $Y$ den Tagesablauf per Telefon und über Facebook (FB) mit der Band. Im Studio treffen $X$ und $Y$ aufeinander, um die Schlagzeugaufnahmen vorzunehmen. Anschließend an das Telefonat (synchrone virtuelle Kopräsenz, reduzierte Kapazitätsbeschränkung) postet der Schlagzeuger auf dem Weg ins Studio in die geschlossene Facebook-Gruppe der Band, dass er den Aufnahmeraum für seine Aufnahmen vormittags nutzt, sodass dieser währenddessen nicht vom Sänger genutzt werden könne. Der Kommunikationskanal Facebook-Gruppe (asynchrone virtuelle Kopräsenz) entspricht einer weiteren reduzierten Kapazitätsbeschränkung, da die Kommunikation in diesem virtuellen Bündel nicht nur keiner physischen Kopräsenz bedarf, sondern auch zeitlich entkoppelt ist. Dennoch treten in diesem virtuellen Bündel neue Autoritäts- und Kapazitätsbeschränkungen auf. Zum einen ist die Facebook-Gruppe der Band geschlossen und nur durch eine persönliche Einladung zugänglich, zum anderen müssen die Mitglieder der Gruppe aufgrund der vielgenutzten virtuellen Kommunikation regelmäßig online verfügbar in response presence sein. Der Produzent und die Band können im Rahmen dieser Verfügbarkeit aller Akteure einen interaktionsbasierten Koordinierungsstil nutzen, wodurch regelmäßige Koordinationstreffen obsolet werden, wie unser Beispiel zeigt: 
Als der Schlagzeuger $Y$ in Abbildung 2 das Studio erreicht, betrachtet der Produzent $X$ den aktuellen Stand des Projektes und antwortet auf das Facebook-Posting des Schlagzeugers, dass heute die physische Anwesenheit des Sängers im Tonstudio nicht erforderlich sei. Die Mitglieder der Band sind den interaktionsbasierten Koordinierungsstil gewohnt, da sie sich auch über den webbasierten Instant-Messenger-Dienst Slack koordinieren. Der Produzent ist in der Slack-Gruppe ausgeschlossen (neue Autoritätsbeschränkungen), damit dort bandinterne Dinge, die auch die Arbeit des Produzenten betreffen, diskutiert werden können. Der Sänger geht davon aus, dass alle das Posting auf Facebook gelesen haben, und schreibt in die Slack-Gruppe, dass er den Proberaum der Band für Gesangsentwicklung verwenden werde, aber trotzdem auf das Feedback des Produzenten angewiesen sei. In dieser Sequenz wird erkennbar, dass beide virtuelle Bündel, die Facebook-Gruppe und die Slack-Gruppe, parallel existieren und für unterschiedliche Zwecke genutzt werden.

Im Studio treffen sich schließlich Produzent $X$ und Schlagzeuger $Y$ in physischer Kopräsenz, um mit den Schlagzeugaufnahmen zu beginnen. Während des Aufnahmeprozesses müssen die Telefone ausgeschaltet oder weggelegt werden, da sie sonst Störgeräusche erzeugen. Dementsprechend sind beide Akteure während der Aufnahme nicht mehr an ihren Handys verfügbar, schauen jedoch in den Pausen regelmäßig auf ihre Handy-Bildschirme. Trotz der laufenden Aufnahmen hat der Produzent am Hauptcomputer die Facebook-Gruppe in einem separaten Fenster ununterbrochen geöffnet. An dieser Sequenz wird deutlich, dass die Nutzung der materiellen Vorrichtungen zur virtuellen Kommunikation in Form eines Telefons (neue Kopplungsbeschränkung) während des Aufnahmeprozesses aufgrund von neuen Autoritätsbeschränkungen (Schutz vor Lärm- und Störgeräuschen) limitiert wird. Trotz der Beschränkung der Handy-Nutzung durch die Schutzmaßnahmen ist eine Nutzung des Computers beinahe zu jeder Zeit möglich, sodass der Zugang zu virtuellen Bündeln innerhalb des Arbeitsprozesses im Studio zu jeder Zeit möglich ist. Die Simultanität von physischen und virtuellen Kopräsenzen im Rahmen von synthetischen Situationen ist die Regel.

Die physische Kopräsenz des Produzenten $X$ und des Schlagzeugers $Y$ im Studio ist notwendig, da die Band mit dem Produzenten entschieden hatte, das Schlagzeug analog aufzunehmen. Diese Entscheidung basiert auf der Tatsache, dass die Band auch live mit analogem Schlagzeug auftritt und die Songs dementsprechend konzipiert sind. Andere Elemente des Albums hingegen werden (auch auf der Bühne) virtuell erzeugt und sind nicht der Anwesenheit im Studio unterworfen. Dennoch werden diese Elemente im Aufnahmeprozess zum Großteil auch in physischer Kopräsenz im Aufenthaltsraum des Studios entworfen einerseits, weil die Expertise des Produzenten dort direkt verfügbar ist, und andererseits, weil die grundsätzliche physische Verfügbarkeit aller Akteure im Schaffensprozess direktes Feedback und vor allem ein gemeinsames Abhören ermöglicht. Ist ein Element fertig, lädt es die Band auf einen geteilten Online-Server hoch, um es für alle beteiligten Akteure verfügbar und bearbeitbar (reduzierte Kopplungsbeschränkung) zu machen. Durch die Virtualisierung des Sounds werden die Musiker raumzeitlich entkoppelt und können sowohl synchron als auch asynchron individuell an den aufgenommenen Versionen arbeiten. Die Entkopplung vom Objekt führte im Falle des Studios dazu, dass der Aufnahmeprozess überwiegend in virtuellen Bündeln durch die Akteure koordiniert wird, wobei der Produzent in seiner Funktion als Experte die Musiker an seine physische Anwesenheit im Studio koppelt.

Physische Anwesenheit im Studio ist - zusammengefasst - notwendig, einerseits für die Aufnahme analoger Instrumente wie Schlagzeug, Gesang, Gitarren und Bässe unter der Expertise des Produzenten, was der klassischen Funktion des Musikstudios entspricht. Andererseits ist das Studio ein abgeschirmter Ort kreativer Auseinandersetzung, innerhalb dessen nicht nur die Entwicklung von Songs sondern parallel dazu, ebenso unter Einfluss des Produzenten, die Aushandlung von Bandidentität und künstlerischen Identitäten geschieht.

\subsection{Komparative Analyse}

Der signifikanteste Unterschied in den Raum-Zeit-Trajektorien der kollaborativen Prozesse in Labor und Studio resultiert aus den jeweils spezifischen Möglichkeiten, die jeweiligen Objekte zu virtualisieren (vgl. Tabelle 3). Arbeiten Akteure im Labor bislang mit einem nur partiell virtualisierbaren Objekt (lebende Zelle), so lässt sich das Objekt im Studio (Sound) grundsätzlich beliebig virtualisieren. Bedingt durch diese unterschiedlichen raumzeitlichen Kopplungs- und Autoritätsbeschränkungen entstehen innerhalb des kollaborativen Prozesses unterschiedliche Anforderungen und Bedingungen für Kopräsenzen.

Zum einen bedingt der Grad der Virtualisierung des Objektes den Zugang zu virtuellen Bündeln zwischen Akteur und Objekt. Ist der Akteur im Labor aufgrund der 
Tabelle 3: Ergebnisse der komparativen Analyse von Labor und Studio

\begin{tabular}{lll}
\hline & Labor & Studio \\
\hline Objekt & Objekt: Zellen & Objekt: Sound \\
& $\begin{array}{l}\text { Grad der Virtualisierung: partiell } \\
\text { virtualisierbar }\end{array}$ & $\begin{array}{l}\text { Grad der Virtualisierung: vollständig } \\
\text { virtualisierbar }\end{array}$ \\
& Starke physische Kopplung an das Objekt & Entkopplung vom Objekt durch Virtualisierung \\
Raumzeitliche Kopplungen & Partielle Entkopplung durch Virtualisierung & Explizite Entscheidung für analoge \\
& der Ergebnisse & Aufnahmepraktiken \\
Koordinierung des Arbeitsprozesses & $\begin{array}{l}\text { Raumzeitliche Koordinierung größtenteils } \\
\text { durch das Objekt und einer Clock-based- }\end{array}$ & $\begin{array}{l}\text { Raumzeitliche Koordinierung größtenteils vom } \\
\text { Pooduzenten und der Band interaktionsbasiert } \\
\end{array}$ \\
\hline
\end{tabular}

nur partiellen Virtualisierung des Objektes physisch stark gekoppelt und sind die Möglichkeiten der virtuellen Bündelung von Akteuren und Objekt stark limitiert, führt die Virtualisierung des Sounds zur raumzeitlichen Entkopplung vom Objekt und ermöglicht virtuelle Bündelungen.

Zum anderen schränken die Schutz- und Sicherheitsvorkehrungen sowie die nur an bestimmten physischen Orten verankerten materiellen Vorrichtungen zur virtuellen Kommunikation die virtuelle Kopräsenz im Labor stark ein, sodass vor allem asynchrone virtuelle Kommunikationsmodi mit einer zeitlich relativ langen Erwiderungsverantwortung (z. B. E-Mail) genutzt werden. Im Studio hingegen, in dem der Zugang zu virtuellen Bündeln fast immer gegeben ist, werden zwar auch überwiegend asynchrone virtuelle Kommunikationsmodi genutzt, jedoch mit einer deutlich kürzeren Erwiderungsverantwortung (z. B. Instant-Messaging-Dienst), sodass es zu einer fast ununterbrochenen response presence kommt. Ein weiterer Unterschied lässt sich in den Koordinierungsstilen beider Arbeitsprozesse finden. Ist der Arbeitsprozess im Labor durch das Objekt und einen Clock-based-Koordinierungsstil stark an raumzeitlich fixierte Aktivität gekoppelt, so ist die Aktivität durch ihre meist gegebene raumzeitliche Entkopplung im Studio deutlich flexibler. Hier übernimmt vor allem der Produzent in seiner Rolle als Experte und seiner physischen Anwesenheit im Studio eine interaktionsbasierte Koordinationsfunktion.

Trotz der sehr unterschiedlichen raumzeitlichen Beschränkungen des kollaborativen Arbeitsprozesses ist beiden erhobenen Raum-Zeit-Trajektorien gemein, dass sowohl das Labor (bedingt durch das Objekt) als auch das Studio (bedingt durch den Produzenten) als physische Anker für physische wie virtuelle kollaborative (Arbeits)praktiken von zentraler Bedeutung sind.

\section{Zusammenfassung und Implikationen}

Ziel unserer methodisch-konzeptionellen Erweiterung ist die Entwicklung eines Instruments zur Konzeption und zur empirisch-qualitativen Erfassung von RaumZeit-Trajektorien in kollaborativen Prozessen. In einem ersten Schritt wurde dazu das zeitgeographische Modell von Hägerstrand um eine virtuelle Dimension erweitert, die nachhaltige Veränderungen der raumzeitlichen Beschränkungen (vgl. Tabelle 1) impliziert. Durch Einbezug virtueller Kopräsenzen (veränderte Kapazitätsbeschränkungen), der Virtualisierung von Objekten (veränderte Kopplungsbeschränkungen) und der virtuellen Interaktionsmedien (veränderte Autoritätsbeschränkungen) können zusätzliche relevante Kontexte (vgl. Tabelle 4) innerhalb des kollaborativen Prozesses erfasst werden.

Mit der Erweiterung der Kapazitätsbeschränkungen um virtuelle Kopräsenzen können insbesondere auch synthetische Situationen innerhalb des kollaborativen Prozesses erhoben werden. Eine Erfassung der raumzeitlichen Kopplung oder Entkopplung zwischen Akteur und Objekt wird durch die Erweiterung der Kopplungsbeschränkungen um virtuelle Objekte ermöglicht. Die Erweiterung der Autoritätsbeschränkungen um virtuelle Mitgliedschaften ermöglicht die Erhebung virtueller Zugangsbeschränkungen zu virtuellen Räumen und Bündel. Zusätzlich zur empirischen Erhebung raumzeitlicher Aktivitätsmuster in kollaborativen Prozessen bietet das erarbeitete Konzept ein methodisches Instrument zur vergleichenden Analyse unterschiedlicher Raum-Zeit-Trajektorien. Ziel dieser Erweiterung war es, einen methodischen Werkzeugkasten zu entwickeln, der kleinste Veränderungen in den Kapazitäts-, Kopplungsund Autoritätsbeschränkungen und ihre Interdependenzen systematisch konzeptualisieren und empirisch 
Tabelle 4: Übersicht der durch die Erweiterung der Zeitgeographie erfassbaren Kontexte

\begin{tabular}{|c|c|c|}
\hline & Erfassbare Kontexte mit Hägerstrand & $\begin{array}{l}\text { Zusätzlich erfassbare Kontexte mit qualitativer } \\
\text { Erweiterung }\end{array}$ \\
\hline Kapazitätsbeschränkungen & Physische Kopräsenzen & Synthetische Situationen, virtuelle Kopräsenzen \\
\hline Kopplungsbeschränkungen & $\begin{array}{l}\text { Kopplung durch physische } \\
\text { Objekteigenschaften }\end{array}$ & $\begin{array}{l}\text { Kopplung/Entkopplung durch Virtualisierung von } \\
\text { Objekten }\end{array}$ \\
\hline Autoritätsbeschränkungen & $\begin{array}{l}\text { Zugangsbeschränkungen zu physischen } \\
\text { Lokalitäten und physischen Bündeln }\end{array}$ & $\begin{array}{l}\text { Zugangsbeschränkungen zu virtuellen Lokalitäten } \\
\text { und virtuellen Bündeln }\end{array}$ \\
\hline
\end{tabular}

abbilden kann. Eine derartige methodische Erweiterung kann jedoch keine inhaltliche Interpretation zum Verlauf von Raum-Zeit-Trajektorien in kollaborativen Prozessen bieten, sondern erhebt lediglich den Anspruch, ein methodisches Werkzeug zur Erfassung und komparativen Analyse raumzeitlicher Aktivitätsmuster in kollaborativen Prozessen bereitzustellen.

In methodischer Hinsicht schlagen wir zur Erhebung der raumzeitlichen Aktivitätsmuster ethnographische Erhebungsstrategien wie beispielsweise teilnehmende Beobachtungen oder Netnographien vor. Der Vorzug der Detailtiefe wird bei diesen Erhebungsstrategien allerdings mit der Herausforderung erkauft, erhebliche Datenmengen (die etwa durch eine Vielzahl synchroner kollaborativer Prozesse im Großraumlabor kontinuierlich generiert werden) analysieren zu müssen. Entsprechende Ansprüche an eine hinreichende Granularität sind vielfach nur über eine Beschränkung auf die Analyse von Teilprozessen einlösbar. Zudem stellt die Virtualisierung und die Synchronität unterschiedlicher Interaktionsmodi in synthetischen Situationen (in der ein Akteur etwa gleichzeitig ein Telefonat führt, On-screen-Projektionen betrachtet und handschriftliche Notizen macht) teilnehmende Beobachtungsstrategien vor grundsätzliche Herausforderungen, die vorläufig nur mit erheblichem Beobachtungsmehraufwand bewältigbar scheinen.

Trotz dieser methodischen Grenzen des entwickelten Instruments konnte mithilfe der Vignetten der RaumZeit-Trajektorien in Labor und Studio gezeigt werden, dass die veränderten raumzeitlichen Bedingungen starken Einfluss auf kollaborative Prozesse haben und die vorgeschlagene qualitativ orientierte Erweiterung des zeitgeographischen Modells um eine virtuelle Dimension für komparative empirische Analysen fruchtbar gemacht werden kann.

\section{Literatur}

Bathelt, H.; Turi, P. (2011): Local, global and virtual buzz: The importance of face-to-face contact in economic interaction and possibilities to go beyond. In: Geoforum 42, 5, 520-529. doi: 10.1016/j.geoforum.2011.04.007

Campos-Castillo, C. (2012): Copresence in Virtual Environments. In: Sociology Compass 6, 5, 425-433. doi: 10.1111/j.17519020.2012.00467.x

Couclelis, H. (2000): From Sustainable Transportation to Sustainable Accessibility: Can We Avoid a New Tragedy of the Commons? In: Janelle, D. G.; Hodge, D. C. (Hrsg.): Information, Place, and Cyberspace: Issues in Accessibility. Berlin, 341-356. doi: 10.1007/978-3-662-04027-0

Couclelis, H. (2004): Pizza over the Internet: e-commerce, the fragmentation of activity and the tyranny of the region. In: Entrepreneurship and Regional Development 16, 1, 41-54. doi: 10.1080/0898562042000205027

Creswell, J. W. (2007): Qualitative inquiry and research design: Choosing among five approaches. London.

Dijst, M. (2004): ICTs and Accessibility: An Action Space Perspective on the Impact of New Information and Communication Technologies. In: Beuthe, M.; Himanen, V.; Reggiani, A.; Zamparini, L. (Hrsg.): Transport Developments and Innovations in an Evolving World. Berlin, 27-46. doi: 10.1007/978-3-540-24827-9

Farber, S.; Neutens, T.; Miller, H.J.; Li, X. (2013): The Social Interaction Potential of Metropolitan Regions: A TimeGeographic Measurement Approach Using Joint Accessibility. In: Annals of the Association of American Geographers 103, 3 , 483-504. doi: 10.1080/00045608.2012.689238

Geertz, C. (1973): Thick Description: Toward an Interpretative Theory of Culture. In: Geertz, C. (Hrsg.): The Interpretation of Cultures. Selected Essays. New York, 310-323.

Girtler, R. (2001): Methoden der Feldforschung. Wien.

Grabher, G. (2002): Cool Projects, Boring Institutions: Temporary Collaboration in Social Context. In: Regional Studies 36, 3, 205-214. doi: 10.1080/00343400220122025

Grabher, G.; Ibert, O. (2014): Distance as asset? Knowledge collaboration in hybrid virtual communities. In: Journal of Economic Geography 14, 1, 97-123. doi: 10.1093/jeg/lbt014

Grabher, G.; Maintz, J. (2007): Learning in personal networks: Collaborative knowledge production in virtual forums. In: Hof, H.; Wengenroth, U. (Hrsg.): Innovationsforschung: Ansätze, Methoden, Grenzen und Perspektiven. Hamburg, 187-202. = Innovationsforschung 1. 
Grabher, G.; Melchior, A.; Schiemer, B.; Schüßler, E.; Sydow, J. (2018): From being there to being aware: Confronting geographical and sociological imaginations of copresence. In: Environment and Planning A 50, 1, 245-255. doi: 10.1177/0308518X17743507

Hägerstrand, T. (1970): What about people in regional science? In: Papers of the Regional Science Association 24, 1, 6-21. doi: 10.1007/BF01936872

Hargadon, A. B.; Bechky, B. A. (2006): When Collections of Creatives Become Creative Collectives: A Field Study of Problem Solving at Work. In: Organization Science 17, 4, 417526. doi: $10.1287 /$ orsc. 1060.0200

Harvey, A.; Macnab, P. A. (2000): Who's up? Global Interpersonal Temporal Accessibility. In: Janelle, D. G., Hodge, D. C. (Hrsg.): Information, Place, and Cyberspace: Issues in Accessibility. Berlin, 147-170. doi: 10.1007/978-3-662-04027-0

Houben, D. (2018): Von Ko-Präsenz zu Ko-Referenz - Das Erbe Erving Goffmans im Zeitalter digitalisierter Interaktion. In: Klemm, M.; Staples, R. (Hrsg.): Leib und Netz. Medienkulturen im digitalen Zeitalter. Wiesbaden, 3-20. doi: 10.1007/978-3658-18863-4_1

Knorr Cetina, K. (2009): The Synthetic Situation: Interactionism for a Global World. In: Symbolic Interaction 32, 1, 61-87. doi: 10.1525/si.2009.32.1.61

Kozinets, R. V. (2009): Netnography: Doing Ethnographic Research Online. London.

Kwan, M.-P. (2000): Interactive geovisualization of activity-travel patterns using three- dimensional geographical information systems: a methodological exploration with a large data set. In: Transportation Research Part C: Emerging Technologies 8, 1-6, 185-203. doi: 10.1016/S0968-090X(00)00017-6

Kwan, M.-P. (2001): Cyberspatial Cognition and Individual Access to Information: The Behavioral Foundation of Cybergeography. In: Environment and Planning B 28, 1, 21-37. doi: 10.1068/ b2560

Kwan, M.-P. (2004): GIS Methods in Time-Geographic Research: Geocomputation and Geovisualization of Human Activity Patterns. In: Geografiska Annaler, Series B: Human Geography 86, 4, 267-280. doi: 10.1111/j.0435-3684.2004.00167.x

Kwan, M.-P. (2007): Mobile Communications, Social Networks, and Urban Travel: Hypertext as a New Metaphor for Conceptualizing Spatial Interaction. In: The Professional Geographer 59, 4, 434-446. doi: 10.1111/j.1467-9272.2007.00633.x

Ling, R. (2004): The Mobile Connection. The cell phone's impact on society. San Francisco.

Maskell, P.; Bathelt, H.; Malmberg, A. (2006): Building global knowledge pipelines: The role of temporary clusters. In: European Planning Studies 14, 8, 997-1013. doi: $10.1080 / 09654310600852332$

Mayring, P. (2008): Qualitative Inhaltsanalyse: Grundlagen und Techniken. Weinheim.

Miller, H. J. (2005): Necessary Space-Time Conditions for Human Interaction. In: Environment and Planning B 32, 3, 381-401. doi: $10.1068 / \mathrm{b} 31154$

Miller, H. J. (2017): Time geography and space-time prism. In: Richardson, D.; Castree, N.; Goodchild, M. F.; Kobayashi, A.; Liu, W.; Marston, R. A. (Hrsg.): The International Encyclopedia of Geography. Sussex, 1-19. doi: 10.1002/9781118786352. wbieg0431 zur Analyse raumzeitlicher Trajektorien in Kollaborationen

Pred, A. (1978): The Impact of Technological and Institutional Innovations on Life Content: Some Time-Geographic Observations. In: Geographical Analysis 10, 4, 345-372.

Schwanen, T. (2007): Matter(s) of interest: artefacts, spacing and timing. In: Geografiska Annaler, Series B: Human Geography 89, 1, 9-22. doi: 10.1111/j.1468-0467.2007.00236.x

Schwanen, T.; Kwan, M.-P. (2008): The Internet, mobile phone and space-time constraints. In: Geoforum 39, 3, 1362-1377. doi: 10.1016/j.geoforum.2007.11.005

Shaw, S.-L.; Yu, H. (2009): A GIS-based time-geographic approach of studying individual activities and interactions in a hybrid physical-virtual space. In: Journal of Transport Geography 17, 2, 141-149. doi: 10.1016/j.jtrangeo.2008.11.012

Thrift, N. (1977): An introduction to time geography. London. = Concepts and Techniques in Modern Geography 13.

Thulin, E.; Vilhelmson, B. (2018): Bringing the background to the fore. Time-geography and the study of mobile ICTs in everyday life. In: Ellegård, K. (Hrsg.): Time Geography in the Global Context: An Anthology. London, 97-112.

Torre, A.; Rallet, A. (2005): Proximity and Localization. In: Regional Studies 39, 1, 47-59. doi: 10.1080/0034340052000320842

Townsend, A. M. (2000): Life in the real-time city: mobile telephones and urban metabolism. In: Journal of Urban Technology 7, 2, 85-104. doi: 10.1080/713684114

Wellman, B. (2001): Physical Place and Cyberplace: The Rise of Personalized Networking. In: International Journal of Urban and Regional Research 25, 2, 227-252.

Yu, H.; Shaw, S.-H. (2008): Exploring potential human activities in physical and virtual spaces: a spatio-temporal GIS approach. In: International Journal of Geographical Information Science 22, 4, 409-430. doi: 10.1080/13658810701427569

Zhao, S. (2003): Toward a Taxonomy of Copresence. In: Presence: Teleoperators and Virtual Environments 12, 5, 445-455.

Zhao, S.; Elesh, D. (2008): Copresence as 'being with'. Social contact in online public domains. In: Information, Communication and Society 11, 4, 565-583. doi: 10.1080/13691180801998995

Zook, M.; Dodge, M.; Aoyama, Y.; Townsend, A. (2004): New Digital Geographies: Information, Communication and Place. In: Brunn, S. D.; Cutter, S. L.; Harrington, J. W. (Hrsg.): Geography and Technology. Dordrecht, 155-176. doi: 10.1007/978-1-40202353-8_7 